a method used for many years in the treatment of migraine and chronic pain, is also effective in PTSD. He claims that $70 \%$ of 2200 people with PTSD in seven US military hospitals were successfully treated with this method. Horner's syndrome (a droopy eyelid) is an unwanted side-effect, but that is a small price to pay for peace of mind free of flashbacks! A group of doctors recently presented data showing evidence of its effectiveness on positron emission tomography, at the American Society of Anesthesiologists' meeting in San Diego, California. Perhaps we should wait to see the published results?

Alkire, M. T., Hollifield, M., Khoshar, R., et al (2015) Neuroimaging suggests that stellate ganglion block improves post-traumatic stress disorder (PTSD) through an amygdala mediated mechanism. At http://www.eugenelipovmd.com; the author has featured in a number of television programmes talking about his findings (http:// globalptsifoundation.org/videos)

\section{Lithium good for the brain and bad/not bad for the kidney?}

T ithium remains the 'gold standard' in the Utreatment of bipolar disorder and the recent finding that it increases grey matter in the brain has given more confidence in its use. However, the fear of long-term damage to the kidneys has been lurking in the background, at least in the minds of the treating clinicians.

The findings of a study carried out in Scotland and published in October 2015 in Lancet Psychiatry offer some reassurance. This was a retrospective, population-based cohort study in patients aged 18-65 who had started maintenance treatment with lithium between 2001 and 2011 and they were compared with a group of patients who had been treated with quetiapine, olanzapine and semi-sodium valproate. The primary outcome was the change in eGFR (estimated glomerular filtration rate) per year, adjusted for age, gender and baseline eGFR; the data were analysed by random coefficient models.

The researchers found no differences between the lithium and non-lithium group and concluded that stable lithium maintenance treatment with levels within therapeutic range is not associated with nephrotoxicity in the absence of acute intoxication.

Clos, S., Rauchhaus, P., Severn, A., et al (2015) Long-term effect of lithium maintenance therapy on estimated glomerular filtration rate in patients with affective disorders: a population-based cohort study. Lancet Psychiatry, 2, 1075-1083.

\section{Stress can lead people to make unhealthy food choices!}

Many people will have made New Year's 1 resolutions about eating more healthily! But we'd be advised to stay away from food when stressed. Maier and colleagues investigated the neural mechanisms involved in how stress may influence self-control. In a study in humans using functional magnetic resonance imaging (fMRI) they found that acute stress made the participants opt for tastier food even if this was less healthy and against their self-imposed diet, compared with the non-stressed control group. This choice pattern was associated with increased functional connectivity between the ventromedial prefrontal cortex (VMPFC) and the amygdala and striatal regions that encode taste. Furthermore, stress was associated with reduced connectivity between the VMPFC and dorsolateral prefrontal cortex, regions linked to self-control. The authors conclude that stress may compromise self-control by enhancing the impact of immediately rewarding attributes and at the same time reducing the efficacy of regions promoting behaviours which are consistent with long-term goals.

Maier, S. U., Makwana, A. B. \& Hare, T. A. (2015) Acute stress impairs self-control in goal-directed choice by altering multiple functional connections within the brain's decision circuits. Neuron, $87,621-631$.

\section{To be or not to be?}

$\mathrm{T}$ he right to be helped to end one's life has been debated for many years and continues to be a hot potato for many countries. The Netherlands, Belgium, Switzerland and others, including some states in the USA, have decriminalised assisted suicide. Many others, such as the UK, continue to debate the issue, which is kept alive as it is repeatedly brought to the public's attention, mostly by people with terminal illness taking their case to court in their wish to prepare for the possibility of euthanasia when the time comes. Euthanasia remains a criminal act for the time being in the UK.

Germany has recently passed a law which brings the subject of assisted suicide to the forefront again. 'Passive euthanasia', such as withholding treatment, has been 'generally accepted' in Germany and there are a number of organisations that provide lethal medications to people who are terminally ill. However, the German parliament has now taken a firm stance and in early November 2015 it voted for a law that criminalises 'commercial euthanasia'. This means that any agency which offers assisted suicide for a fee will be prosecuted. What about assisted suicide offered free of charge? The German law needs to be clarified.

What about the rest of us - should we be free to choose to be 'euthanased' or not? The debate goes on...

\section{Correction}

In the November 2015 issue of BJPsych International (vol. 12, no. 4), the surname of the fifth author of the Chile country profile (p. 95) should have been spelt Vanegas (not Venegas). The online version has been corrected, post-publication and in deviation from print 